cause a greater force is brought to bear on the perinæal structures. In cases of the kind now under consideration, the attention of the practitioner will be most profitably employed in ascertaining why it is that the head of the child is not propelled downwards with greater rapidity on the perinæum. In many of these cases of so-called rigidity of the perinæum, a careful use of the forceps is the best, the proper remedy, the uterine contractions being deficient in power.

The case which I have, in the course of the series of practical observations now brought to a conclusion, argued, is the one of rational versus empirical treatment of the perinæum during labour. I think there can be little doubt as to the nature of the decision, which will be formed by those who take the pains to consider the question attentively and dispassionately.

36, Berkcley Square, W., A pril 1861.

\section{olitiginal Communitations.}

\section{CASE OF DEFERRED LACTATION.}

By Thomas Skinner, M.D., Obstetrical Physician to the Liverpool Dispensaries.

I was lately called to a lady who had just had a severe rigor, and was threatened with inflammation of one or both breasts. The patient, who was the mother of several children, told me that she had had a miscarriage in Scotland about two months before, and that she had every reason to believe she was at the time in the seventh month of pregnancy. At the time of my visit, I detected a little milk in the breasts, and my patient informed me that the week before the occurrence of the "weed", she felt the usual sensation of a " rush of milk" to the breasts, and that milk was then secreted in abundance.

It is interesting to observe, that the date of these latter phenomena corresponds to what would have been the week following the full time of her late gestation. The lady had not been nursing, nor was it known that there was any milk in the breasts from the time of the miscarriage until the week before the rigor.

\section{STRANGULATED OBLIQUE INGUINAL HERNIA IN A FEMALE: OPERA- TION : SUCCESSFUL RESULT.}

By George Lawson, F.R.C.S., Surgeon to the Great Northern Hospital, Clinical Assistant to the Royal Ophthalmic Hospital, Moorfields.

THE occurrence of strangulated inguinal hernia in women is so rare, that each case claims for itself a special amount of interest. The following are the partreulars of a case which has lately been under my care :

Mary E., aged 56, married, the mother of one child, for the last ten years had suffered from an oblique inguinal hernia of the left side, which, she said, had been in the habit of coming down frequently. For the last three years she had worn a truss; but it fitted her badly, and often, when on, allowed the bowel to descend.

On April : 2 d, the hernia came down as low as the beft labium at about five o'clock in the afternoon. All her endeavours to return it failed, and she, consequently, applied to Mr. Dickinson, who used the taxis and the other usual remedies, but without success. Abdominal pain of a severe twisting character quickly came on, accompanied by severe vomiting.
At 12.30 A.sr., seven hours from the descent of the bowel, and the commencement of the symptoms of strangulation, I saw her. 'The tumour was then of about the size of a large egg, very tense and tender, and occupied directly the site of the inguinal canal. Failing to return the bowel under the influence of chloroform, I at once operated. It proved to be an oblique inguinal heruia ; and, as a division of the external ring gave no relief, and as the sac was much thickened and adherent to the surrounding tissues, I opened it, when the seat of the stricture was found at its neck. The bowel was immediately returned, but the sac was left in situ, and the skin closed over it. An opiate was given soon after the operation. The patient progressed most favourably: and, without the use of any purgatives, her bowels were freely opened on the eighth and ninth days after the operation.

April 24. She is now quite well.

\section{Transactions of 迶ramethes.}

\section{EAST YORK AND NORTH LINCOLN BRANCH.}

CANCER OF THE PENIS: AMPUTATION : RECOVERY.

By JoHN MORLEY, Esq., Barton-on-Humber.

$$
\text { [Read September } 26 t h, 1860 \text {.] }
$$

THE subject of cancer presenting itself so often to our notice in its various forms and in different organs, although not so frequently (according to $\mathrm{my}$ limited experience) in the penis as in other parts, and the serious mutilation necessary for its removal, as in the following example, form my apology for bringing before my professional brethren what might at first sight appear to be a very simple case, but upon a correct diagnosis and treatment of which depended the retention or loss of the important organ involved.

I am not aware that I have anything novel to com. municate; but, as I have been so ably assisted in the microscopical examination and illustration of the diseased parts by Dr. Munroe, I gladly avail myself of this opportunity of bringing the case before you, as an instance of the benefit to be derived from association in the cultivation of everything relating to our noble profession. The readiness with which the required help was rendered, deserves my warmest thanks; and I hope that the recital of the following case will draw forth from other members information on the subject, which will tend to advance our knowledge of the pathology and treatment of cancer in general.

CASE. William Cook, aged 51, a pig.jobber, residing at Barton, married, first consulted me on May 1st, 1858, on account of a tumour situated on the internal aspect of the prepuce, which had latterly caused him a consi. derable amount of pain. He was the subject of congenital phimosis. He had syphilis about thirty years ago; since which period the inguinal glands had remained slightly enlarged. About five years ago, he observed what appeared to him to be a small wart near the extremity of the prepuce, which was attended with itching and smarting, and gradually enlarged to its pre. sent state. The extremity of the penis was inflamed and enlarged; and it was impossible to denude the glans; a hard tumour, however, could be detected by raising the skin between the finger and thumb.

On consultation with my friend Mr. Eddie (who also lindly assisted me at the subsequent operations) it was decided to excise the diseased portion of prepuce, which was done on May $6 \mathrm{th}$. The glans at this time presented a mottled appearance (red and white) on the surface opposed to the preputial tumour. Water dressing was applied at first, until the rapid growtb of fungous granu489 